\title{
A simple, inexpensive, liquid-tight swivel connection for use in chronic infusion systems
}

\author{
MILTON A. TRAPOLD \\ University of Minnesota, Minneapolis, Minnesota 55455
}

\begin{abstract}
This note describes the fabrication of a simple, inexpensive, liquid-tight swivel joint for twist-free coupling of small, freely moving animals to infusion systems. The device uses a disposable hypodermic needle and syringe as the main components, and fabrication requires only readily available hand tools and minimal mechanical expertise.
\end{abstract}

In various experimental preparations, it is desirable to connect subjects to an infusion pump or similar liquid-delivery device for extended periods of time. A common problem with such preparations, especially when the subject is free to move about in its environment, involves rotational movement of the subject with respect to the tubing that connects the subject to the pump. Such rotation typically causes the tubing to twist, kink, and eventually to pinch off, thereby obstructing further delivery of liquid to the subject until the tubing has been untwisted. The untwisting is often accomplished by manually disconnecting the tubing, removing the twists, and then reconnecting. This requires continuous surveillance of the subjects, and the procedures involved in disconnecting the tubing may seriously interfere with other experimental procedures.

One effective method of minimizing the problem of twisted infusion tubing is to insert a liquid-tight swivel joint into the tubing line. This prevents transmission of torque from the subject to the tubing. Epstein and Teitelbaum (1962) have described one very effective design for a liquid-tight swivel that can be modified easily to accommodate a variety of tubing sizes, types of tubing connections, liquid viscosities, delivery rates, and so on (cf. Gross, Trapold, \& Hyde, 1968). However, fabrication of the Epstein-Teitelbaum type of device requires fairly sophisticated shop facilities and a substantial amount of time, even for an experienced machinist.

This note describes an alternative design for a liquid-tight swivel that can be constructed in a few minutes using commonly available hand tools and a few cents worth of parts. The two major components

This research was supported in part by grants to the Center for Research in Human Learning from the National Science Foundation (BHS-75-03816) and the National Institute of Child Health and Human Development (HO-01136). Reprints may be obtained from Milton A. Trapold, Department of Psychology, Elliott Hall, 75 E. River Road, Minneapolis, Minnesota 55455. required in fabrication of this device are a plastic disposable hypodermic syringe and a hypodermic needle. The component sizes described here were found to be very satisfactory for a specific application using rats. Several dimensions of the device can be modified easily to adapt it to other applications. The procedure for constructing the device is as follows.

\section{FABRICATION PROCESS}

(1) Completely remove the plunger assembly from the barrel of a 3-cc disposable hypodermic syringe. Then remove the rubber tip from the plunger by twisting it off of its mounting knob on the end of the plastic plunger shaft. Keep the rubber plunger tip and the syringe barrel; discard the plastic plunger shaft.

(2) Push a 1-in. or longer hypodermic needle through the center of the rubber plunger tip, entering the hole into which the plastic plunger had fit and exiting in the center of the surface of the tip that normally would push against the liquid in the syringe. It is important that the needle go through the tip as close to the exact center of the tip as possible. Push the rubber tip as far back onto the shaft of the needle as possible without binding or otherwise restricting the freedom of the needle to rotate in the hole it has pierced through the rubber tip. It is this rotation of the needle shaft in the rubber tip that will provide the liquid-tight swivel action of the completed device.

(3) With wire cutters, remove one bead link from a length of $.5-\mathrm{cm}$ diam bead chain (the type of chain commonly found in key chains and readily available in hardware stores). Thread this metal bead onto the needle shaft until it barely touches the rubber tip, and solder the end of the bead that is away from the rubber tip to the needle shaft. This soldering can be facilitated by the use of stainless steel soldering flux (commonly available at hardware stores or machinist suppliers), and by vigorously abrading the surfaces to be soldered with a file or emory cloth. The purpose of this bead is to prevent the needle shaft from pulling 
out of the rubber tip. After soldering, thoroughly rinse the needle, bead, and rubber tip assembly to remove any excess flux.

(4) With wire cutters, cut the needle shaft off $.8 \mathrm{~cm}$ below the soldered end of the metal bead. Dress off the crimp caused by this cutting by touching the tip of the needle to a fine grinding wheel, or by rubbing it on a file, emory cloth, or whetstone. This dressing leaves a visible burr around the lumen of the needle, which can most easily be removed by inserting the point of a suitably smaller (e.g., 25-gauge) hypodermic needle into the lumen of the dressed needle and rotating a few times. Alternatively, any other appropriately sized instrument or wire that can be forced into the needle lumen will also remove most of the burr.

(5) With a razor blade or a sharp knife, cut the barrel of the 3-cc plastic syringe off $1.8 \mathrm{~cm}$ from the zero mark on the syringe. Keep the section of the barrel that contains the needle fitting. Discard the remainder of the barrel.

(6) Insert the needle, bead, and rubber tip assembly into the syringe barrel (see Figure 1) as far as possible without restricting the freedom of rotation of the needle.

(7) Using a drill press, a power hand drill, or a hand-twist drill, make a small hole through both sides of the syringe barrel immediately above the top of the rubber tip. The purpose of this hole is to allow insertion of a retaining pin through the barrel to lock the needle assembly in place inside the barrel. This retaining pin can be a piece of wire, a brad, a small cotter pin, or anything else that will provide a solid barrier against the needle assembly pulling out of the barrel. Bus (19-gauge) wire works very well. It is stiff enough to perform the retaining function, but flexible enough to be bent easily with pliers to cotter it into place as it exits from the syringe barrel (see Figure 1), or to provide additional clearance for the needle shaft inside the barrel (see below).

In order that the retaining pin not inhibit rotation of the needle shaft inside the barrel, the retaining-pin hole

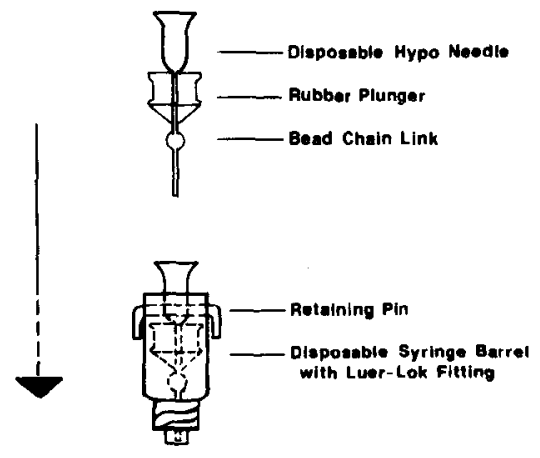

Figure 1. Schematic representation of construction of liquidtight swivel (see text for details). should be drilled about $.3 \mathrm{~cm}$ off center on the syringe barrel.

(8) Insert the retaining pin, making sure that it is locked securely in place either by bending it as it exits from the barrel, or by use of friction fit in the retainingpin hole. Check to make sure that the retaining pin does not rub against the needle shaft or hub, since this will inhibit free rotation of the needle. If the pin does rub against the needle, the situation can usually be corrected by forcing needle-nosed pliers into the barrel and bending the pin away from the needle. Alternatively, a more suitably placed retaining-pin hole easily can be drilled.

\section{DISCUSSION}

A device constructed as described here has an overall length of $3.5 \mathrm{~cm}$, a maximum width of $1.0 \mathrm{~cm}$, a female Luer fitting on the input end (the needle hub), and a male Luer fitting on the output end (the syringe output fitting). This arrangement has worked very well with a chronic nasogastric preparation patterned after that described by Snowden (1975) but modified so that the connection at the animal is a standard hypodermic needle hub affixed to the animal's skull with dental acrylic (Gross et al., 1968). Into this needle hub is plugged the male Luer output end of the device described here. To make this connection with the animal more secure, swivels are constructed from disposable syringes having Luer-Lok tips. The use of syringes having Luer friction connection tips is preferable when frequent connection and disconnection of the swivel is required.

The input end of the swivel is connected directly to the tubing that comes from an infusion pump. This is either commercial hypodermic extension tubing that comes prefit with a plastic male Luer fitting on one end and a female Luer fitting on the other end, or it is general-purpose tubing to which Luer-Lok tubing connectors are appended.

This type tubing connector can also be used to modify the output of the swivel to fit into preparations, such as that described by Snowden (1975), that terminate in a short length of plastic into which hypodermic tubing is force fit.

Disposable 21-gauge needles work very well in this device, and they cost only a small fraction of the price of "nondisposable" needles. The selection of 21 gauge is largely arbitrary. Larger or smaller gauge needles work equally well, although needles larger than 19 gauge require the existing hole through the metal bead chain link to be enlarged before it will accept the needle shaft, and needles smaller than 23 gauge are lacking in rigidity. A 3-cc syringe has proven to be an ideal size for our application, although exactly the same principles and fabrication steps will work with any size disposable syringe.

A large number of these devices have been used to 
connect nasogastric rats to an infusion pump on an around-the-clock basis, and no serious problems have been encountered. The liquid-tight fepature of this device has seldom failed, even on occasions in which the nasogastric tubing on the animal became occluded, resulting in very high liquid pressure inside the swivel. There has been an occasional problem with friction-only Luer fittings working loose, but this is easily circumvented by using all Luer-Lok fittings.

The cost of these devices is trivial. Syringes and needles are purchased in lots of 100 from a local hospital supply vendor for about 3 cents each. Bead chain links from local hardware stores figure out to about .3 cents each. With a little practice, fabrication of one of these devices takes less than $5 \mathrm{~min}$ and requires only a few hand tools and practically no previous experience in a shop.

\section{REFERENCES}

Epstein, A. N., \& Teitelbaum, P. A watertight swivel joint permitting chronic injections into animals. Journal of Applied Physiology, 1962, 17, 171-172.

Gross, D. M., Trapold, M. A., \& Hyde, T. S. A simple technique for delivering liquids directly into the mouth of an unrestrained rat. Journal of Experimental Analysis of Behavior, 1968, 11, 191-195.

SNOWdEN, C. T. Gastrointestinal manipulation in the rat. In D. Singh \& D. D. Avery (Eds.), Physiological techniques in behavioral research. Monterey: Brooks Cole, 1975.

(Received for publication August 24, 1978; revision accepted October $31,1978$. ) 